Research paper

\title{
Prophylactic intranasal administration of a TLR2/6 agonist reduces upper respiratory tract viral shedding in a SARS-CoV-2 challenge ferret model
}

\author{
Pamela C. Proud ${ }^{1,6}$, Daphne Tsitoura ${ }^{2,6}$, Robert J. Watson ${ }^{1}$, Brendon Y. Chua ${ }^{3}$, Marilyn J. Aram ${ }^{1}$, \\ Kevin R. Bewley ${ }^{1}$, Breeze E. Cavell ${ }^{1}$, Rebecca Cobb ${ }^{1}$, Stuart Dowall ${ }^{1}$, Susan A. Fotheringham ${ }^{1}$, \\ Catherine M.K. Ho ${ }^{1}$, Vanessa Lucas ${ }^{1}$, Didier Ngabo ${ }^{1}$, Emma Rayner ${ }^{1}$, Kathryn A. Ryan ${ }^{1}$, \\ Gillian S. Slack ${ }^{1}$, Stephen Thomas ${ }^{1}$, Nadina I. Wand ${ }^{1}$, Paul Yeates ${ }^{1}$, Christophe Demaison ${ }^{2}$, \\ Weiguang Zeng ${ }^{3}$, Ian Holmes ${ }^{2}$, David C. Jackson ${ }^{3}$, Nathan W. Bartlett ${ }^{4}$, Francesca Mercuri ${ }^{2,7, *}$, \\ Miles W. Carroll ${ }^{1,5,7, *}$

\footnotetext{
${ }^{1}$ National Infection Service, Public Health England (PHE), Porton Down, Salisbury, Wiltshire, United Kingdom SP4 0JG

${ }^{2}$ Ena Respiratory, Level 9, 31 Queen St, Melbourne, Victoria, 3000, Australia

${ }^{3}$ Department of Microbiology and Immunology, The University of Melbourne, at The Peter Doherty Institute for Infection and Immunity, 792 Elizabeth St, Melbourne, Victoria 3000, Australia

${ }^{4}$ Viral Immunology and Respiratory Disease group and Priority Research Centre for Healthy Lungs, University of Newcastle and Hunter Medical Research Institute, Newcastle, Australia
} \\ ${ }^{5}$ Nuffield Dept of Medicine, Oxford University, Oxford, UK
}

\section{A R T I C L E I N F O}

\section{Article History:}

Received 24 August 2020

Revised 16 October 2020

Accepted 13 November 2020

Available online $\mathrm{xxx}$

\section{Keywords:}

Ferret

COVID-19

SARS-CoV-2

Viral shedding

TLR-2

INNA-051

\begin{abstract}
A B S T R A C T
Background: The novel human coronavirus SARS-CoV-2 is a major ongoing global threat with huge economic burden. Like all respiratory viruses, SARS-CoV-2 initiates infection in the upper respiratory tract (URT). Infected individuals are often asymptomatic, yet highly infectious and readily transmit virus. A therapy that restricts initial replication in the URT has the potential to prevent progression of severe lower respiratory tract disease as well as limiting person-to-person transmission.

Methods: SARS-CoV-2 Victoria/01/2020 was passaged in Vero/hSLAM cells and virus titre determined by plaque assay. Challenge virus was delivered by intranasal instillation to female ferrets at $5.0 \times 10^{6} \mathrm{pfu} / \mathrm{ml}$. Treatment groups received intranasal INNA-051, developed by Ena Respiratory. SARS-CoV-2 RNA was detected using the 2019-nCoV CDC RUO Kit and QuantStudio ${ }^{\mathrm{TM}} 7$ Flex Real-Time PCR System. Histopathological analysis was performed using cut tissues stained with haematoxylin and eosin (H\&E).

Findings: We show that prophylactic intra-nasal administration of the TLR2/6 agonist INNA-051 in a SARSCoV-2 ferret infection model effectively reduces levels of viral RNA in the nose and throat. After 5 days postexposure to SARS-CoV-2, INNA-051 significantly reduced virus in throat swabs $(p=<0.0001)$ by up to a 24 fold ( $96 \%$ reduction) and in nasal wash $(p=0.0107)$ up to a 15 fold (93\% reduction) in comparison to untreated animals.

Interpretation: The results of our study support clinical development of a therapy based on prophylactic TLR2/ 6 innate immune activation in the URT, to reduce SARS-CoV-2 transmission and provide protection against COVID-19.

Funding: This work was funded by Ena Respiratory, Melbourne, Australia.

(C) 2020 The Authors. Published by Elsevier B.V. This is an open access article under the CC BY-NC-ND license
\end{abstract} (http://creativecommons.org/licenses/by-nc-nd/4.0/)

\footnotetext{
* Corresponding author.

E-mail addresses: fm@enarespiratory.com (F. Mercuri), miles.carroll@phe.gov.uk (M.W. Carroll).

6 These authors contributed equally.

${ }^{7}$ Joint Corresponding and senior authors.
}

\section{Introduction}

Coronaviruses (CoV) are pleomorphic, positive-sense, singlestranded RNA-enveloped viruses, members of the Coronoviridae family, that mainly infect wild animals and cause mild human disease [1]. In addition, seven human CoVs that belong to either the Alphacoronavirus- or Betacoronavirus-genus have now been identified. Four of these human CoVs usually cause a mild, upper respiratory 


\section{Research in context}

\section{Evidence before the study}

Toll Like Receptors (TLR), the sentinel stimulators of the host immune defence against invading microbes, are recognised as targets for the development of broad-spectrum antivirals. Ena Respiratory's candidate TLR2/6 agonists, including INNA-051, had been shown to reduce virus levels in the upper respiratory tract and lungs in mouse challenge models of rhinovirus and influenza. When the COVID-19 pandemic was declared, we raised the question "Would priming the innate immunity at the site of infection with a TLR2/6 agonist reduce SARS-Cov- 2 virus levels and be of potential use to stop disease progression and community transmission?" We searched PubMed for articles in English before 23 March 2020, using the search terms "“"SARSCoV-2" OR "COVID-19") AND ("TLR agonists') in all fields. We found no original research articles. To answer this question, a collaboration was set up between Ena Respiratory and Public Health England, who had established a SARS-CoV-2 virus challenge model in ferrets.

\section{Added value of this study}

Our study provides evidence that a TLR2/6 agonist delivered topically to the respiratory tract is highly effective against SARS-CoV-2 (COVID-19) in the ferret challenge model, reducing viral levels in the nose and throat of treated animals by up to $96 \%$. The results of this study support the clinical rationale of using a TLR2/6 agonist as prophylaxis, to reduce SARS-CoV-2 transmission and to protect against COVID-19 disease progression.

\section{Implications of all the available evidence}

Vaccination is the most attractive approach for long-term protection against SARS-CoV-2 and other viral respiratory infections that cause serious health complications and spread quickly and widely in the community. In a global push to create SARS-CoV-2 vaccines, there are concerns that vaccines in clinical development might have limited ability to reduce viral shedding and virus transmission in the community, as well as limited efficacy in at risk populations such as elderly individuals. There remains an immediate and pressing need for complementary approaches to stop viral community transmission and disease progression. Adjunct to effective social distancing, face masks and vaccine approaches, priming the innate immunity at the site of infection with a pharmaceutical agent, such as INNA051, is a promising approach to fight SARS-CoV-2, particularly to health care providers, vulnerable individuals and compromised patient groups.

tract illness (common cold). Another three novel human CoVs have emerged in the past two decades through transmission to humans via an intermediate animal host [2], and caused outbreaks of significant respiratory morbidity and mortality: in 2003, Severe Acute Respiratory Syndrome (SARS) CoV in China [3], in 2012 Middle Eastern Respiratory Syndrome (MERS) CoV in Saudi Arabia [4] and in December 2019 a novel CoV, SARS-CoV-2, identified in the lower respiratory tract of patients presenting viral pneumonia in Wuhan, China [5]. Unlike the highly pathogenic SARS or MERS CoVs, SARS$\mathrm{CoV}-2$ infections have spread rapidly around the globe, causing broad spectrum respiratory symptoms, from very mild to severe, lifethreatening disease (COVID-19) mostly in at risk populations such as the elderly and those with comorbidities.
As with other respiratory CoVs, SARS-CoV-2 primarily spreads via the airborne route, with respiratory droplets expelled by infected individuals [6]. Virus can be transmitted from symptomatic, as well as pre- or asymptomatic individuals [7,8], with asymptomatic individuals being able to shed virus, and therefore being capable to transmit the disease, for longer than those with symptoms [9]. As with other respiratory viruses such as influenza, recent evidence suggests that, the epithelium of the upper respiratory tract (URT) is the initial site of SARS-CoV-2 infection [10,11]. This is consistent with the abundant nasal epithelial cell expression of the SARS-CoV-2 receptor, angiotensin-converting enzyme 2 (ACE2) and its decreasing expression throughout the lower respiratory tract [11].

A topical treatment of the URT that boosts anti-viral immunity and restricts viral replication is a promising method to promote viral clearance, reduce viral shedding and transmission. The TLRs are key microbe-recognition receptors with a crucial role in activation of host defence and protection from infections and therefore attractive drug targets against infectious diseases [12-14]. Synthetic agonists of the intracellular viral DNA/RNA-recognising TLR molecules, TLR3, TLR7/8 and TLR9, are capable of boosting protective innate immune responses against respiratory viruses [15]. However, their success in the clinic has been limited, due to short-duration of benefit or induction of adverse effects, related to the release of pro-inflammatory cytokines and activation of the type-1 Interferon pathway [16-19]. TLRs expressed on the cell surface such as TLR2 offer an alternative approach. TLR2 dimerizes with TLR1 or TLR6 to recognize a broad variety of commensal and pathogenic microbial molecules and its activation is tightly regulated to maintain immune homeostasis [20]. A series of novel synthetic molecules, named the INNA compounds, have been developed with TLR2/6 agonist properties. Importantly, TLR2/6 agonists of the INNA compound series do not directly activate Type- 1 interferons (unpublished data).

Airways administration of INNA compounds has been shown to protect from lethal influenza virus infection, prevent viral transmission and secondary bacterial superinfections in mouse disease models [21-23]. Intranasal (i.n.) treatment with INNA compounds also reduces viral load and lung inflammation in mouse models of rhinovirus infection (unpublished data). The demonstrated prophylactic benefit is associated with fast TLR2/6-mediated up-regulation of a series of innate immune response elements in airway epithelial cells, defined by early, rapid expression of NF- $\kappa$ B-regulated anti-microbial genes, including IFN- $\lambda$ and chemokines, that precede immune cell recruitment and support prolonged antiviral defence, suppresses viral load and virus-induced pulmonary inflammation (unpublished data).

Ferret challenge models are commonly used to understand human respiratory virus-induced diseases and to evaluate the efficacy of related vaccines and drugs [24,25]. Use of ferrets is appropriate in the case of SARS-CoV-2 infection, as they express the virus entry ACE2 receptor in their airways [26-28] and SARS-CoV-2 i.n. inoculation in ferrets results in virus replication in the URT and dosedependent viral shedding $[28,29]$.

To determine whether TLR2/6 agonists are also active against SARS-CoV-2, we used prophylactic i.n. administration of the novel compound INNA-051, in a SARS-CoV-2 challenge ferret model [29].

\section{Methods}

\subsection{Animals and ethics}

Twenty-four healthy, female outbred ferrets (Mustela putorius furo) aged 6-8 months were obtained from a UK Home Office accredited supplier. The mean weight at the time of first INNA-051 treatment was $845 \mathrm{~g} /$ ferret (range 740-1040 g). Animals were housed in social groups of six prior to and post INNA-051 treatment at Advisory Committee on Dangerous Pathogens (ACDP) containment level 2. 
Group sizes of 6 ferrets were used to satisfy the UK Home Office approved project licence requirements for reduction, but to allow determination of statistical significance between groups. Animals were transferred to ACDP containment level 3 and housed in pairs post SARS-CoV-2 challenge. Cages met with the UK Home Office Code of Practice for the Housing and Care of Animals Bred, Supplied or Used for Scientific Procedures (December 2014). Access to food and water was ad libitum and environmental enrichment was provided. Animals were sedated by intramuscular injection of ketamine/xylazine $(17.9 \mathrm{mg} / \mathrm{kg}$ and $3.6 \mathrm{mg} / \mathrm{kg}$ bodyweight) for administering of treatments, in-life sampling and viral challenge.

All experimental work was conducted in accordance with and under the authority of a UK Home Office approved project licence that had been subject to local ethical review at PHE Porton Down by the Animal Welfare and Ethical Review Body (AWERB) as required by the Home Office Animals (Scientific Procedures) Act 1986.

\subsection{INNA-051 treatment}

INNA-051 belongs to a series of closely-related, pegylated synthetic analogues of the diacylated lipopeptide, S-[2,3-bis(palmitoyl oxy)propyl] cysteine ( $\mathrm{Pam}_{2} \mathrm{Cys}$ ) (INNA compound series), with selective TLR2/TLR6 agonist activity. Pam ${ }_{2}$ Cys is inherently insoluble and has been rendered soluble by others through addition of the amino acid motif SK4 [30]. Oligo lysine sequences have, however, been shown to be toxic, albeit at high concentration [31] and to modulate viral infection processes independent of TLR activation [32]. Any offtarget effects were mitigated by incorporating polyethylene glycol as a solubilising agent, in the INNA compound series [32]. The $\mathrm{EC}_{50} \mathrm{~S}$ for INNA-051 for the human TLR2/6 receptor is calculated at $40.1 \mathrm{pg} / \mathrm{mL}$ or $\sim 19 \mathrm{pM}$.

Freeze dried INNA-051 provided by Ena Respiratory, Melbourne, Australia was resuspended in phosphate buffered saline (PBS) (1 mg/ $\mathrm{ml}$ ) and stored $2-8{ }^{\circ} \mathrm{C}$. Immediately prior to treatment, INNA-051 $(1 \mathrm{mg} / \mathrm{ml}$ ) was further diluted in PBS to the required treatment doses; high dose $(100 \mu \mathrm{g} / \mathrm{ml})$, low dose $(20 \mu \mathrm{g} / \mathrm{ml})$ and mixed dose $(20 \mu \mathrm{g} /$ $\mathrm{ml}$ first dose and $100 \mu \mathrm{g} / \mathrm{ml}$ second dose).

\subsection{Viral challenge inoculum}

SARS-CoV-2 Victoria/01/2020 [33] was generously provided by Peter Doherty Institute for Infection and Immunity, Melbourne, Australia at P1 and passaged twice in Vero/hSLAM cells [ECACC Cat\# 04091501, RRID:CVCL_L037], obtained from the European Collection of Authenticated Cell Cultures (ECACC) PHE, Porton Down, UK. Whole genome sequencing was performed, on the challenge isolate, SISPA protocol and then sequenced using Nanopore as described previously [34]. Virus titre was determined by plaque assay on Vero/E6 cells [ECACC Cat\# 85020206, RRID:CVCL_0574]. Challenge substance dilutions were conducted in Phosphate Buffer Saline (PBS). Challenge virus was delivered by intranasal instillation $(1.0 \mathrm{ml}$ total, $0.5 \mathrm{ml}$ per nostril) at $5.0 \times 10^{6} \mathrm{pfu} / \mathrm{ml}$.

\subsection{Experimental design}

Experimental design and viral challenge dose were informed by a previous dose-dependent ferret study [29]. Prior to commencing the experiment, animals were randomly assigned to the four treatment groups, to minimise bias. Assignment was not based on weight or any other notable characteristics. To assign animal IDs to groups, ID numbers were randomised by assigning a value to each using RAND() function in Excel and ordering them low to high. A temperature/ID chip (Bio-Thermo Identichip, Animalcare Ltd, UK) was inserted subcutaneously into the dorsal cervical region of each animal.

INNA-051 was delivered by intranasal instillation $(1.0 \mathrm{ml}$ total, $0.5 \mathrm{ml}$ per nostril) to three groups $(n=6)$ of ferrets 4 days and 1 day prior to challenge. On each day, group 1 received a high dose [100 $\mu \mathrm{g} / \mathrm{ml}$ ], group 2 a low dose [20 $\mu \mathrm{g} / \mathrm{ml}]$ and group 3 received a $20 \mu \mathrm{g} / \mathrm{ml}$ dose 4 days prior to challenge and a $100 \mu \mathrm{g} / \mathrm{ml}$ ] dose 1 day before challenge. PBS was delivered to control group ferrets $(n=6)$ 4 days and 1 day prior to challenge. Two ferrets each from the high dose, low dose and control groups were scheduled for euthanasia on day $3(n=6)$ to assess pathology at early stage infection. Remaining ferrets $(n=18)$ were scheduled for euthanasia on days $12-14$; high and low dose [day $12 n=1$, day $13 n=2$, day $14 n=1$ ], mixed dose [ $n=2$ days $12-14$ ] and control [ $n=2$ days 12 and 14$]$.

Nasal washes and throat swabs for all ferrets were taken prior to first treatment, at days 1 and 3 pc $(n=24)$ and at days 5, 7, 10 and 12 pc for surviving ferrets $(n=18)$. At necropsy, tissue samples were taken for histopathology and analysed by PCR. Nasal washes were obtained by flushing the nasal cavity with $2 \mathrm{ml}$ PBS. Cotton throat swabs (Koehler Technische Produkten, VWR) were gently stroked across the back of the pharynx in the tonsillar area and retained in viral transport media (VTM). Throat swabs were processed, and aliquots were stored in AVL at $-80{ }^{\circ} \mathrm{C}$ until assay.

\subsection{Clinical and euthanasia observations}

Animals were monitored for clinical signs of disease twice daily (approximately 8 hours apart) for the entirety of the experiment. Clinical signs of disease were assigned a score based upon the following criteria. Activity was scored as follows; $0=$ alert and playful, 1 = alert, playful when stimulated, 2 = alert, not playful when stimulated, 3 = not alert or playful. No clinical signs were noted throughout the experiment. To meet the requirement of the project license, immobility, neurological signs or a sudden drop in temperature were predetermined automatic euthanasia criteria. Animals were also deemed to have reached a humane endpoint if their body weight was at or below $30 \%$ baseline. If any ferret reached any of these three criteria, they were to be immediately euthanised using a UK Home Office approved Schedule 1 procedure. No animals reached these end-points during this study.

Temperature was taken using a microchip reader and implanted temperature/ID chip. Temperature was recorded at each clinical scoring point using the chip to ensure any peak of fever was recorded. Animals were weighed at the same time each day throughout the experiment.

\subsection{Necropsy procedures}

Ferrets were anaesthetised with ketamine/xylazine $(17.9 \mathrm{mg} / \mathrm{kg}$ and $3.6 \mathrm{mg} / \mathrm{kg}$ bodyweight) and exsanguination was effected via cardiac puncture, followed by injection of an anaesthetic overdose (sodium pentabarbitone Dolelethal, Vetquinol UK Ltd, $140 \mathrm{mg} / \mathrm{kg}$ ). A necropsy was performed immediately after confirmation of death. The left lung was dissected and used for subsequent virology procedures.

\subsection{RNA extraction}

RNA was isolated from nasal wash, throat swabs and lung tissue. Weighed lung tissue was homogenised and inactivated in RLT (Qiagen) supplemented with $1 \%(\mathrm{v} / \mathrm{v})$ Beta-mercaptoethanol. Tissue homogenate was then centrifuged through a QIAshredder homogenizer (Qiagen) and supplemented with ethanol as per manufacturer's instructions. Non-tissue samples were inactivated in AVL (Qiagen) and ethanol. Downstream extraction on all inactivated samples was then performed using the BioSprint ${ }^{\mathrm{TM}} 96$ One-For-All vet kit (Indical) and Kingfisher Flex platform as per manufacturer's instructions. 


\subsection{Quantification of Viral Loads by RT-qPCR}

Reverse transcription-quantitative polymerase chain reaction (RTqPCR) targeting a region of the SARS-CoV-2 nucleocapsid (N) gene was used to determine viral loads and was performed using TaqPath $^{\mathrm{TM}}$ 1-Step RT-qPCR Master Mix, CG (Applied Biosystems ${ }^{\mathrm{TM}}$ ), 2019-nCoV CDC RUO Kit (Integrated DNA Technologies) and QuantStudio $^{\mathrm{TM}} 7$ Flex Real-Time PCR System. Sequences of the N1 primers and probe were: 2019-nCoV_N1-forward, 5' GACCCCAAAATCAGCGAAAT 3'; 2019-nCoV_N1-reverse, 5' TCTGGTTACTGCCAGTTGAATCTG 3'; 2019-nCoV_N1-probe, 5' FAM-ACCCCGCAT TACGTTTGGTGGACC-BHQ1 3'. The cycling conditions were: $25^{\circ} \mathrm{C}$ for $2 \mathrm{~min}, 50^{\circ} \mathrm{C}$ for $15 \mathrm{~min}, 95^{\circ} \mathrm{C}$ for $2 \mathrm{~min}$, followed by 45 cycles of $95^{\circ}$ $\mathrm{C}$ for $3 \mathrm{~s}, 55^{\circ} \mathrm{C}$ for $30 \mathrm{~s}$. The quantification standard was in vitro transcribed RNA of the SARS-CoV-2 N ORF (NCBI Reference Sequence: NC_045512.2) with quantification between $1 \times 10^{1}$ and $1 \times 10^{6}$ copies $/ \mu 1$. Positive samples detected below the lower limit of quantification (LLOQ) were assigned the value of 5 copies $/ \mu \mathrm{l}$, whilst undetected samples were assigned the value of $<2.3$ copies $/ \mu 1$, equivalent to the assays lower limit of detection (LLOD).

\subsection{Histopathological analysis}

Samples from the left cranial and left caudal lung lobe together with nasal cavity, were fixed by immersion in 10\% neutral-buffered formalin and processed routinely into paraffin wax. Nasal cavity samples were decalcified using an EDTA-based solution prior to embedding. $4 \mu \mathrm{m}$ sections were cut and stained with haematoxylin and eosin (H\&E) and examined microscopically. In addition, samples were stained using the RNAscope technique to identify the SARSCoV-2 virus RNA. Briefly, tissues were pre-treated with hydrogen peroxide for $10 \mathrm{~min}$ (room temperature), target retrieval for $15 \mathrm{~min}$ $\left(98-101{ }^{\circ} \mathrm{C}\right)$ and protease plus for $30 \mathrm{~min}\left(40{ }^{\circ} \mathrm{C}\right.$ ) (Advanced Cell Diagnostics). A V-nCoV2019-S probe (Cat No. 848561, Advanced Cell Diagnostics) was incubated on the tissues for $2 \mathrm{~h}$ at $40{ }^{\circ} \mathrm{C}$. Amplification of the signal was carried out following the RNAscope protocol using the RNAscope 2.5 HD Detection kit - Red (Advanced Cell Diagnostics).

\subsection{Statistical analysis}

Statistical analyses were performed using GraphPad Prism, version 8.4.2 (GraphPad Software, Inc., San Diego, CA). No data was excluded from analysis. Weight change from baseline was compared for groups 1-4 using a Kruskal-Wallis test comparing area under the curve (AUC). Transformed viral titre values fitted to a straight line in a QQ plot confirming normal distributions for comparison. Viral titres were compared from each of the treated groups versus control group 4 by two-way ANOVA with Dunnett's multiple comparisons test. Viral titres from groups 1-3 combined were compared against control group 4 by two-way ANOVA with Sidak's multiple comparisons test. In each test a p-value $<0.05$ was considered statistically significant.

\subsection{Role of the funding source}

The funders, Ena Respiratory, contributed to study design, interpretation, writing of the manuscript and the decision to publish.

\section{Results}

\subsection{Clinical observations in ferrets}

Ferrets received two i.n. administrations of INNA-051 or vehiclecontrol (PBS), at 4 days before and 1 day prior to i.n. challenge with $5.0 \times 10^{6}$ plaque-forming units (PFU) of SARS-CoV-2 in $1 \mathrm{ml}$ volume (day 0). Four groups (6 animals/group) were used: Group 1 received two $1 \mathrm{ml}$ doses (100 $\mu \mathrm{g} / \mathrm{mL}$ ) INNA-051 (high dose); Group 2 received two $1 \mathrm{ml}$ doses (20 $\mu \mathrm{g} / \mathrm{mL}$ ) INNA-051 doses (low dose); Group 3 received one $1 \mathrm{ml}$ dose $(20 \mathrm{ug} / \mathrm{mL}$ ) INNA-051 at -4 days and one $1 \mathrm{ml}$ dose INNA-051 (100 $\mu \mathrm{g} / \mathrm{mL})$ at -1 day (mixed dose); Group 4 received two doses of vehicle (PBS) alone. After inoculation with SARS-CoV-2, ferrets were monitored for 12 days. In life samples were taken at days $1,3,5,7,10$ and 12 , with scheduled culls at days 3 $(n=6)$ and end of study days $12-14(n=18)$ (Fig 1A).

Previous in vivo studies in mice have shown that respiratory application of INNA compounds have a good safety profile, without significant pro-inflammatory side effects or systemic cytokine release syndrome. Intranasal administration to ferrets of two doses of INNA051, prior to SARS-CoV-2 challenge, did not induce observable or measurable clinical signs of inflammation, or changes in the animal's activity.

Assessment of body temperature revealed some variation between treatment groups (Fig. 1B), with 2 of 6 ferrets in the INNA051 high dose group 1 showing a transient increase of temperature $>40.0^{\circ} \mathrm{C}$, only after the first, but not the second dose and 3 of 6 ferrets in group 3 showing transient temperature increase $>40.0^{\circ} \mathrm{C}$ after only the second, higher dose. It is common for natural ferret diurnal temperatures to rise to $40{ }^{\circ} \mathrm{C}$ and with no other clinical signs or changes in behaviour observed, it is difficult to interpret if this transient rise is clinically meaningful. Animals progressively gained weight through the course of the study across all groups, with no difference in body weight between groups at any time (Fig 1C). Analysis of area under the curve using a Kruskal-Wallis test showed no significant difference between groups.

It has been previously described that SARS-CoV-2 infection in ferrets is not associated with the development of severe symptomatology, but it represents a robust model of mild disease that allows the study of respiratory viral replication $[29,35]$. In this context, no obvious differences in clinical disease signs were observed among treatment groups in this experimental study. Assessment of body temperature (Fig. 1B) and weight loss (Fig. 1C) did not reveal significant variation between the INNA-051 and PBS-treated groups.

\subsection{INNA-051 reduces viral load following SARS-CoV-2 challenge}

To follow the dynamics of SARS-CoV-2 viral replication and assess the impact of INNA-051 prophylactic treatment, nasal wash and throat swab samples were taken 4 days before viral challenge and at $1,3,5,7,10$ and 12-days post challenge (dpc). Analysis of viral RNA in nasal wash samples at $1 \mathrm{dpc}$ confirmed infection in all treatment groups, with lower viral RNA levels detected in INNA-051 treatment Group 3 (Fig. 2A). Reduction of viral RNA in treatment Group 3 was also evident at $3 \mathrm{dpc}(p=0.0155)$ (Fig. 2A). By $5 \mathrm{dpc}$. all INNA-051 treated groups had significantly reduced viral RNA compared to the vehicle-control group (2-way ANOVA Dunnett's multiple comparison test: Group $1 p=0.0244$; Group $2 p=0.0107$; Group $3 p=0.0071$ compared to vehicle-control Group 4) (Fig 2A). On $5 \mathrm{dpc}$, the viral RNA levels in the nasal washes of the majority of INNA-051 treated animals remained low or below quantifiable limits throughout the course of infection. Viral RNA levels were found to be below the level of quantification in nasal washes of PBS-treated animals from $10 \mathrm{dpc}$ onwards (Fig 2A).

Analysis of viral RNA in throat swabs provided further evidence of the capacity of INNA-051 treatment to reduce SARS-CoV-2 in the URT (Fig 2B). On 3 dpc lower viral RNA levels were found in throat swabs of INNA-051 treated animals, with significantly greater reduction observed ( $p=0.0345,2$-way ANOVA Dunnett's multiple comparison) in INNA-051 treatment Group 3. By $5 \mathrm{dpc}$, all groups treated with INNA-051 had significantly reduced viral RNA levels, compared to the vehicle control group (2-way ANOVA Dunnett's multiple comparison: Group $1 p=0.0002$, Group $2 p=<0.0001$ and Group $3 p=0.0039$ compared to vehicle control Group 4). Highly significantly reduction 
a

SARS-CoV-2

challenge

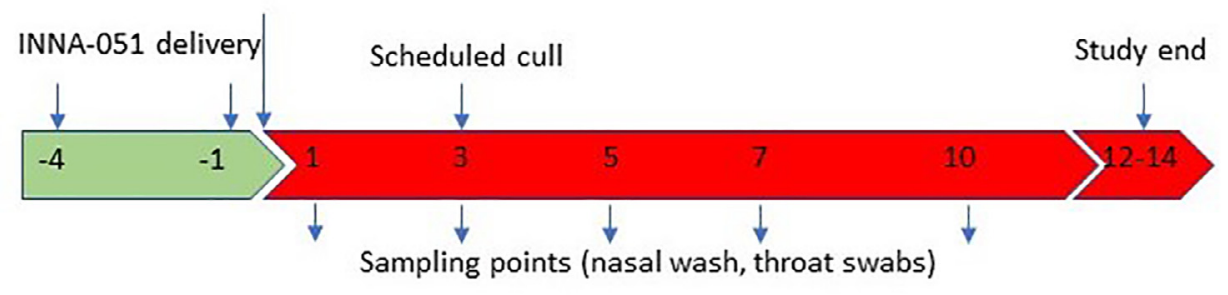

b

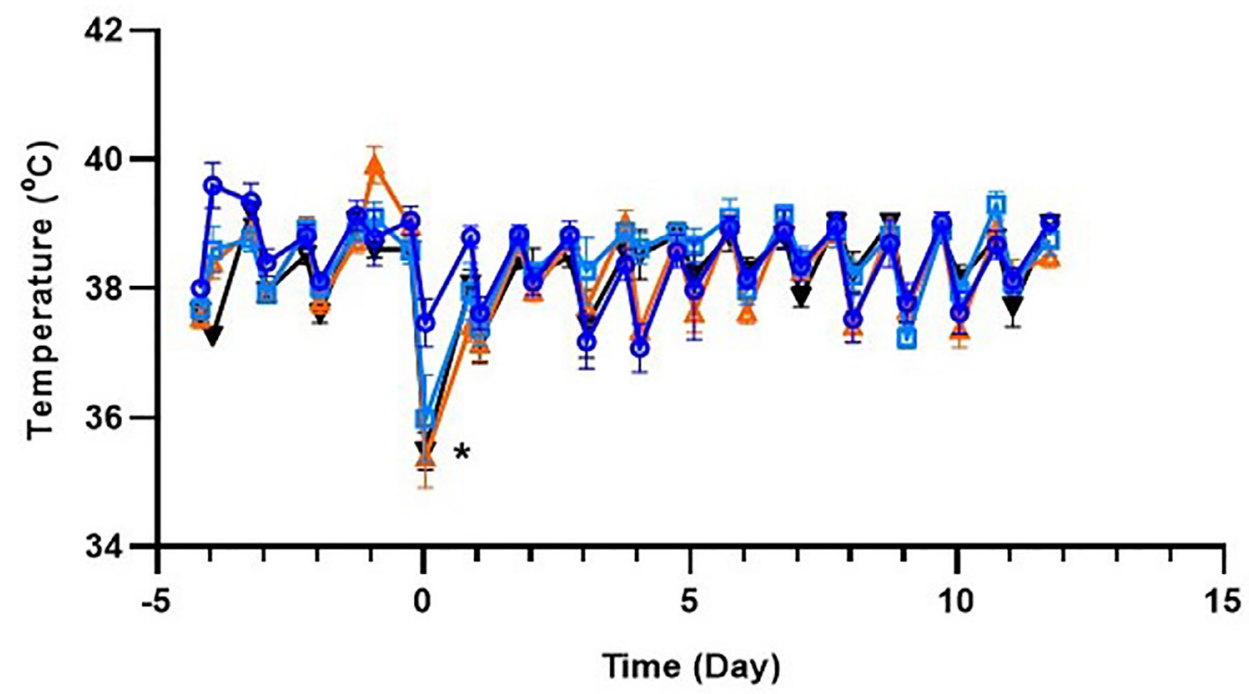

C

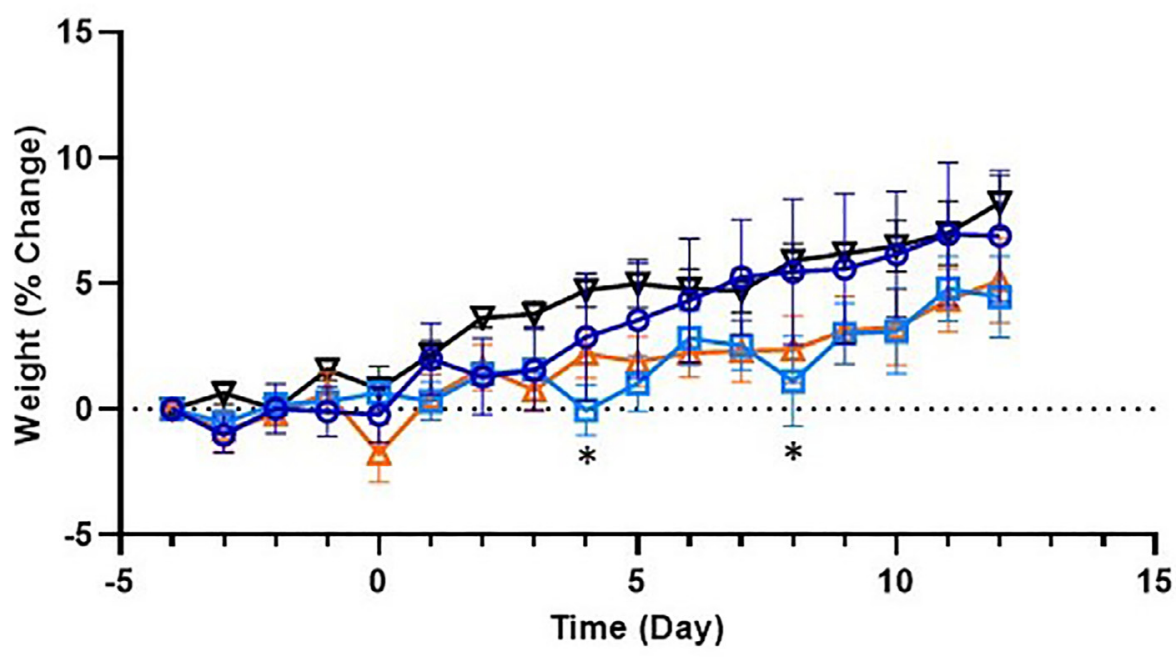

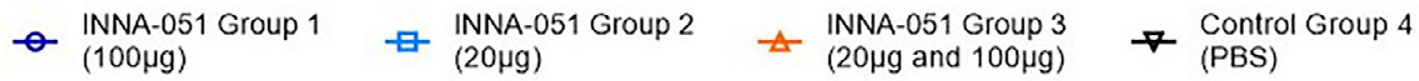

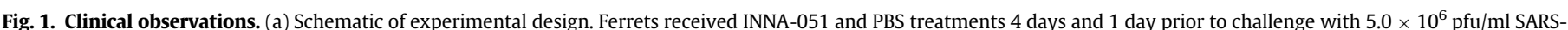

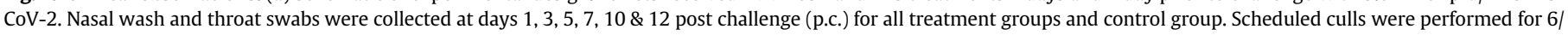

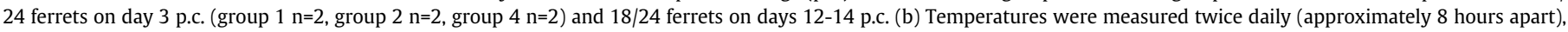

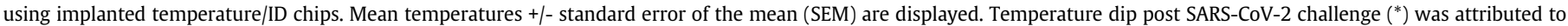

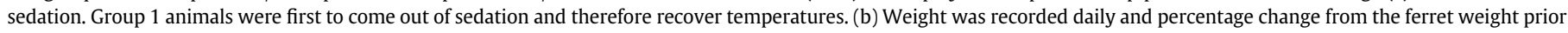

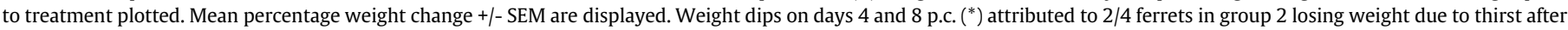
upturning water bowl overnight. Ferrets in group 3 (2/6 animals) lost weight for same reason on day 8 p.c. 


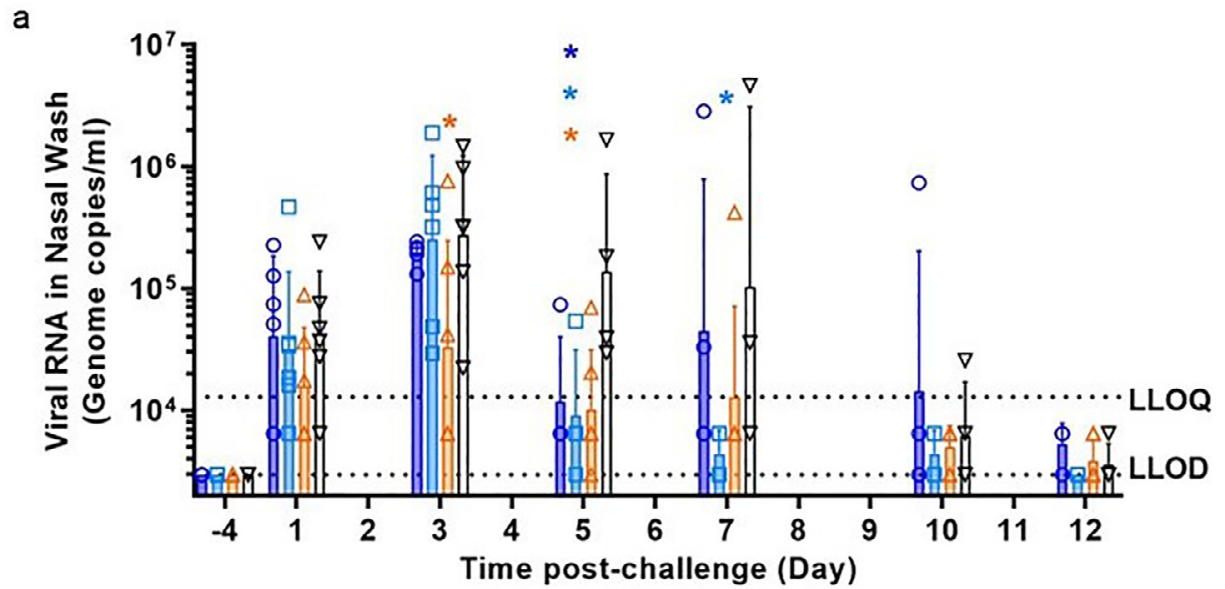

b

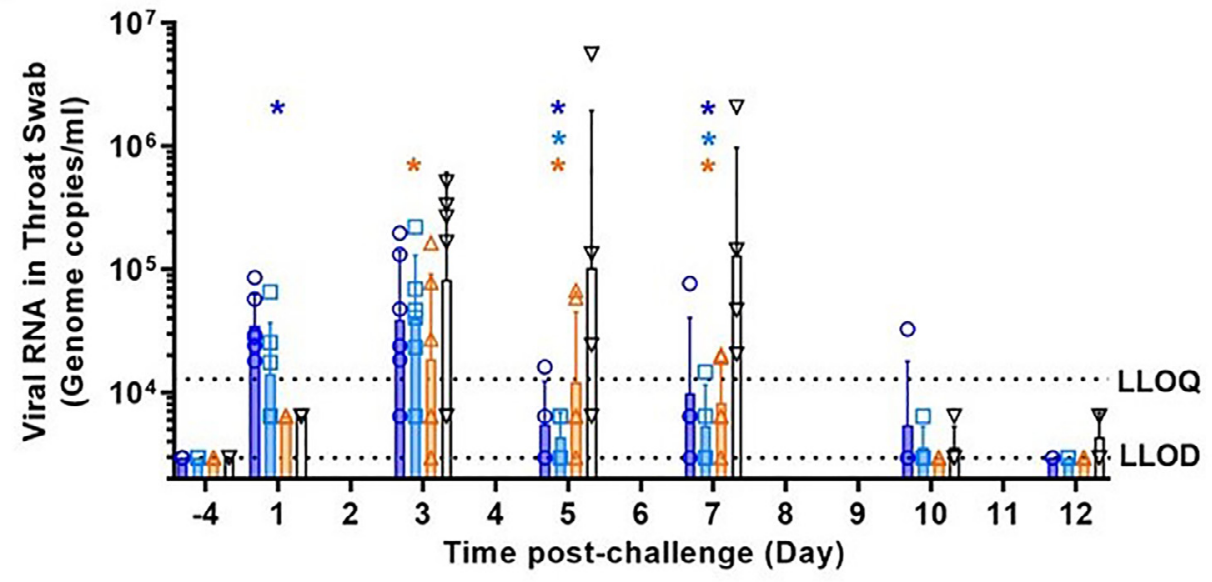

C

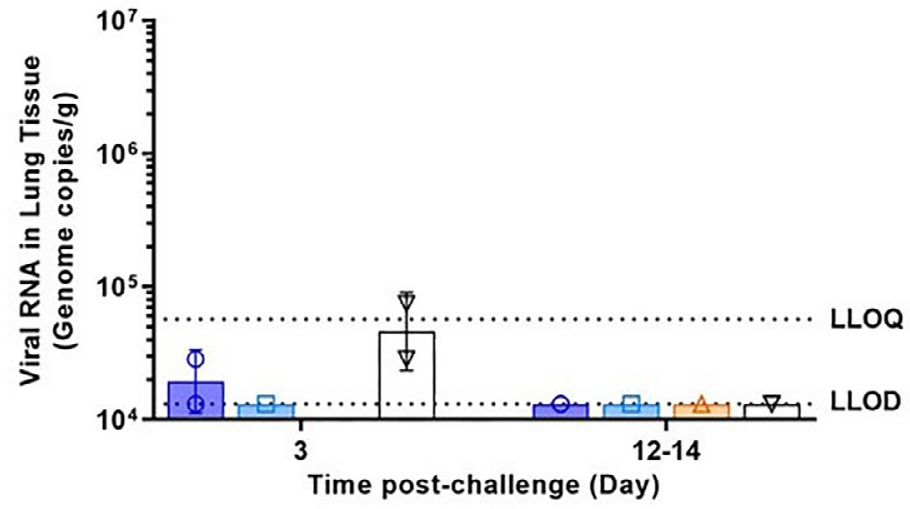
- INNA-051 Group 1

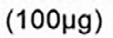
INNA-051 Group 2
$(20 \mu g)$
INNA-051 Group 3
$(20 \mu \mathrm{g}$ and $100 \mu \mathrm{g})$
$\nabla \quad$ Control Group 4
(PBS)

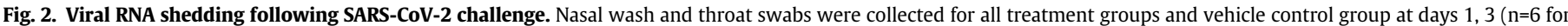

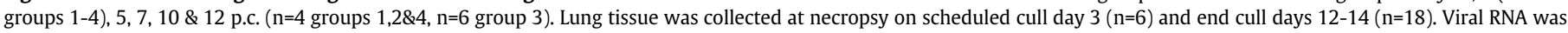

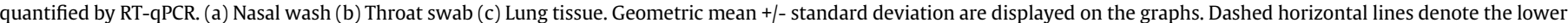

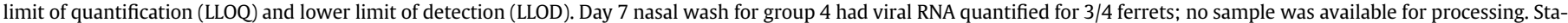

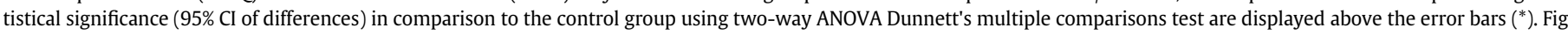

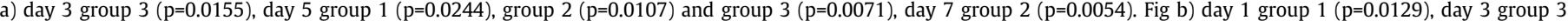
$(\mathrm{p}=0.0345)$, day 5 group $1(\mathrm{p}=0.0002)$, group $2(\mathrm{p}=<0.0001)$ and group $3(\mathrm{p}=0.0039)$, day 7 group $1(\mathrm{p}=0.0014)$, group $2(\mathrm{p}=<0.0001)$ and group $3(\mathrm{p}=0.0002)$.

in viral RNA in the throat of INNA-051 treated animals was also apparent on $7 \mathrm{dpc}$ (2-way ANOVA Dunnett's multiple comparison: Group $1 p=0.0014$, Group $2 p=<0.0001$ and Group $3 p=0.0002$ compared to vehicle control Group 4), while by $10 \mathrm{dpc}$ to the end of the study, the levels of viral RNA were below the limit of quantitation in all treatment groups (Fig 2B).

Variation in viral titres of individual animals within groups is an expected outcome of using outbred ferrets and can be seen in a 
similar ferret challenge experiments [29]. Additional analysis performed using Pearson's correlation shows that the correlation between viral tires in the nose and throat for the individual animals is highly significant at days 5 and 7 p.c (supplementary figures).

Because all INNA-051 treatment groups exhibited reduced viral RNA in the nose and throat, we combined these groups into a single data set (supplementary figures) and compared to the group treated with vehicle. Using 2-way ANOVA Sidak's multiple comparison test, significant reduction in nasal viral RNA was observed at $5 \mathrm{dpc}$ $(p=0.0057)$ and highly significant $(p<0.0001)$, greater than 10 -fold reduction in throat viral RNA was apparent from 5 to $7 \mathrm{dpc}$ following INNA-051 i.n. treatment (Figure S2). After 5 days post-exposure to SARS-CoV-2, animals treated with INNA-051 in group 2 had statistically significant reduction of virus in throat swabs $(p=<0.0001,2$ way ANOVA Sidak's multiple comparison test) with 24 fold (96\% reduction) and nasal wash ( $p=0.0107,2$-way ANOVA Sidak's multiple comparison test) 15 fold ( $93 \%$ reduction) in this group compared to untreated animals.

These results indicate a similar profile with the protective effects of natural acquired immunity in the ferret challenge model, as observed following re-challenge with SARS-CoV-2 [29].

To assess SARS-CoV-2 detected beyond the URT, lung tissue samples were collected, on scheduled cull day 3 (6/24 animals) and days $12-14$ (18/24 animals) dpc and analysed for viral RNA levels. On day $3 \mathrm{dpc}$, two culled ferrets from the control vehicle group had detectable viral RNA levels $\left(7.42 \times 10^{4}\right.$ and $2.86 \times 10^{4}$ copies $\left./ \mathrm{ml}\right)$ (Fig 2C). There was one ferret in Group 1 showing detectable, but below the quantifiable limit, viral RNA, and no other INNA-051 treated ferrets showing detectable viral RNA in lung tissue on day 3 and days $12-14$ dpc.

\section{Discussion}

This study provides evidence supporting a novel approach to prevent SARS-CoV-2 transmission, based on reduced viral shedding, following prophylactic i.n administration of INNA-051. Global efforts for prevention of SARS-CoV-2 infection have so far been mostly focused on social distancing and hygiene measures as well as on R\&D efforts for the development of vaccines. Our data demonstrate, for the first time, in an in vivo SARS-CoV-2 infection model, that INNA-051 is highly effective at reducing URT viral shedding, providing the potential to control virus transmission and COVID-19 disease.

TLR2 stimulation at mucosal surfaces triggers rapid up-regulation of protective, innate immune defence responses, and also activates counter-regulatory signalling that suppresses development of excessive inflammation and tissue damage and promotes the integrity of local epithelial barrier function $[36,37]$. In addition, the INNA compounds have been specifically designed to exert TLR2-mediated pharmacological activity on mucosal epithelium, without being systemically absorbed (Ena Respiratory unpublished data), a property that is expected to facilitate their development as safe, antiviral drug candidates. The lack of obvious clinical signs of inflammation following the administration of two doses of INNA-051 administered i.n supports this view. Histopathology from the study indicates that i.n. INNA-051 administration does not exacerbate SARS-CoV-2 pathology in the ferret lung in this setting (Figure S3).

It has been previously shown that i.n administration of an INNA compound in a mouse model of influenza triggers a cascade of innate immune signals that results in reduction of viral load, prevention of lower-respiratory infection and viral transmission between animals [21-23].

Although the ferret SARS-CoV-2 model has limitations and may not represent the severe spectrum of COVID-19 disease, our findings are highly encouraging and indicative of the potential impact i.n. administration of INNA-051 prophylactically may have against SARS-
CoV-2 in humans. Though the prophylactic effects of INNA-051 showed statistical significance across all INNA-051 treated groups, further work is needed to determine the optimal dosing. The fact that a significant reduction of URT viral RNA levels was observed in INNA-051-treated outbred ferrets during the peak of viral replication (5-7 days dpc) in this model [29] implies airway immunity priming and enhancement of antiviral host defence. The predictive value of antiviral effectiveness data from respiratory viral infection ferret models and translation into human infectious disease has been established $[25,38]$. For this reason, the SARS-CoV-2 ferret model has been used, during the current pandemic, to evaluate the therapeutic effect of a number of FDAapproved/repurposed drugs including, lopinavir-ritonavir, hydroxychloroquine sulfate, or emtricitabine-tenofovir [39]. These drugs were found to have no or only modest ( 4 fold for emtricitabinetenofovir) effect against SARS-CoV2 viral replication, as measured by viral titres in nasal wash from the ferrets [39].

Substantial reduction of SARS-CoV-2 viral shedding in the URT and therefore control of respiratory virus transmission may not be easily achievable without potentiation of airways antiviral immune defences [40]. Systemic antiviral drugs, as well as vaccines may not be effective in halting respiratory viral transmission even if they achieve suppression of clinical disease and in fact preliminary results from an experimental study with one of the leading SARS-CoV-2 vaccine candidates (an adenovirus-construct expressing SARS-CoV-2 spike protein) in non-human primates have shown little effect on the virus load in nasal washes [41]. To address these potential limitations, particularly during the urgent circumstances of an epidemic, parallel use of an i.n. administered innate immune modulator with the characteristics of INNA-051 may be highly appropriate to rapidly boost innate immunity at the primary site of respiratory infection which is protective within days of treatment. The use of i.n. INNA-051 for antiviral respiratory prophylaxis therefore offers several additional advantages, including fast-acting protection, and is in contrast to vaccines that take $2-4$ weeks to mount a protective response. The limited risk for development of antiviral resistance, the option of self-administration and the non-prohibitive cost for large-scale manufacturing are also especially attractive factors.

In conclusion, this study provides evidence that prophylactic $i$. $\mathrm{n}$. administration of the TLR2/6 agonist INNA-051 offers a promising approach for prevention and management of SARS-CoV-2 infection that can be used as a stand-alone method of antiviral prophylaxis and is complimentary to potential vaccination programs. This approach is particularly appealing to individuals at elevated risk of community transmission or development of severe disease, including front-line health care workers, vulnerable communities, the elderly, the immunocompromised and those with existing comorbidities.

\section{Funding sources}

This work was funded by Ena Respiratory, Melbourne, Australia.

\section{Contributors}

N.W.B, B.Y.C, C.D, D.C.J, F.M, P.P, D.T and M.W.C. contributed to the concept and design of the study. P.P and D.T. wrote the manuscript. N.W.B, B.Y.C, C.D, S.D, D.C.J, F.M. and M.W.C. provided critical review. P.P co-ordinated the study and performed analysis on data generated. J.D. and M.G.C. provided virus strain. I.H, D.C.J and W.Z contributed to the design and preparation of the novel compound used in this study. K.R.B. grew viral stock, provided challenge material and assisted with statistical analysis. S.A.F and P.Y. managed all animal procedures. B.E. C, R.C, C.M.K.H, V.L, D.N, K.A.R. and S.T. contributed to the processing of all animal samples at containment level 3. E.R directed histology 
experiments and performed critical assessment of pathology. M.J.A, N.I.W and R.J.W. performed the extraction and PCR of samples. G.S performed quality control and analytical assistance on PCR data. All authors have read and approved the final version of the manuscript.

\section{Declaration of Competing Interests}

Authors report grants from Ena Respiratory, during the conduct of the study. W. Zeng and D.C. Jackson reports grants from Ena Therapeutics, during the conduct of the study. D. Tsitoura, C. Demaison, F. Mercuri, I. Holmes and N.W. Bartlett reports personal fees and other from Ena Therapeutics, outside the submitted work. D.C. Jackson, W. Zeng and B.Y. Chua reports other from Ena Therapeutics, outside the submitted work. Dr. Holmes reports personal fees from Ena Therapeutics, outside the submitted work.

In addition, D. Tsitoura, C. Demaison and F. Mercuri have a patent AU 2020901709 pending to Ena Therapeutics. D.C Jackson, W. Zeng and C. Demaison have a patent PCT/AU2011/001225 issued to Ena Therapeutics. D.C Jackson, W. Zeng, I. Holmes and C. Demaison have a patent PCT/AU2020/050660 pending to Ena Therapeutics.

\section{Acknowledgements}

The authors gratefully acknowledge the support from the Biological Investigations Group at the National Infection Service, PHE, Porton Down, United Kingdom. Special thanks to Thomas Hender, Carrie Turner, Stephen Findlay-Wilson and Neil McLeod for assisting in providing RT-qPCR data for this work. The authors would like to express their gratitude to Jade Gouriet, Phillip Brown, Karen Gooch and Jemma Paterson for their help in processing of Containment Level 3 in vivo samples. The authors would like to thank Laura Hunter, Chelsea L. Kennard and Francisco J. Salguero for their contribution to the processing and critical review of pathology tissues. The authors are grateful to Michael G. Catton and Julian Druce from Victorian Infectious Disease Reference Laboratory for the generous donation of the SARS-CoV-2 strain. The authors gratefully acknowledge Ena Respiratory for funding this work.

\section{Data sharing statement}

The data that support the findings of this study are available on request from the corresponding author for Ena Respiratory, F.M, upon signing a material transfer agreement. The data are not publicly available due to animal study ethical restrictions.

\section{Supplementary materials}

Supplementary material associated with this article can be found, in the online version, at doi:10.1016/j.ebiom.2020.103153.

\section{References}

[1] Cui J, Li F, Shi Z-L. Origin and evolution of pathogenic coronaviruses. Nat Rev Microbiol 2019;17(3):181-92.

[2] Li X, Zai J, Zhao Q, Nie Q, Li Y, Foley BT, et al. Evolutionary history, potential intermediate animal host, and cross-species analyses of SARS-CoV-2. J Med Virol 2020;92(6).

[3] Zhong NS, Zheng BJ, Li YM, Poon LLM, Xie ZH, Chan KH, et al. Epidemiology and cause of severe acute respiratory syndrome (SARS) in Guangdong, People's Republic of China, in February, 2003. Lancet North Am Ed 2003;362(9393):13538.

[4] Zaki AM, van Boheemen S, Bestebroer TM, Osterhaus ADME, Fouchier RAM. Isolation of a novel coronavirus from a man with pneumonia in Saudi Arabia. N Engl J Med 2012;367(19):1814-20.

[5] Lu R, Zhao X, Li J, Niu P, Yang B, Wu H, et al. Genomic characterisation and epidemiology of 2019 novel coronavirus: implications for virus origins and receptor binding. Lancet North Am Ed 2020;395(10224):565-74.
[6] Tay MZ, Poh CM, Rénia L, MacAry PA, Ng LFP. The trinity of COVID-19: immunity, inflammation and intervention. Nat Rev Immunol 2020;20(6):363-74.

[7] Hu Z, Song C, Xu C, Jin G, Chen Y, Xu X, et al. Clinical characteristics of 24 asymptomatic infections with COVID-19 screened among close contacts in Nanjing, China. Sci China Life Sci 2020;63(5):706-11.

[8] Zou L, Ruan F, Huang M, Liang L, Huang H, Hong Z, et al. SARS-CoV-2 viral load in upper respiratory specimens of infected patients. N Engl J Med 2020;382 (12):1177-9

[9] Long Q-X, Tang X-J, Shi Q-L, Li Q, Deng H-J, Yuan J, et al. Clinical and immunological assessment of asymptomatic SARS-CoV-2 infections. Nat Med 2020.

[10] Richard M, van den Brand JMA, Bestebroer TM, Lexmond P, de Meulder D, Fouchier RAM, et al. Influenza A viruses are transmitted via the air from the nasal respiratory epithelium of ferrets. Nat Commun 2020:11(1):766.

[11] Hou YJ, Okuda K, Edwards CE, Martinez DR, Asakura T, Dinnon 3rd KH, et al. SARS$\mathrm{CoV}-2$ reverse genetics reveals a variable infection gradient in the respiratory tract. Cell 2020

[12] Hennessy EJ, Parker AE, O'Neill LA. Targeting toll-like receptors: emerging therapeutics? Nat Rev Drug Discovery 2010;9(4):293-307.

[13] Hedayat M, Netea MG, Rezaei N. Targeting of toll-like receptors: a decade of progress in combating infectious diseases. Lancet Infect Dis 2011:11(9):702-12.

[14] Patel MC, Shirey KA, Pletneva LM, Boukhvalova MS, Garzino-Demo A, Vogel SN, et al. Novel drugs targeting toll-like receptors for antiviral therapy. Future Virol 2014;9(9):811-29.

[15] To EE, Erlich J, Liong F, Luong R, Liong S, Bozinovski S, et al. Intranasal and epicutaneous administration of Toll-like receptor 7 (TLR7) agonists provides protection against influenza A virus-induced morbidity in mice. Sci Rep 2019;9(1):2366.

[16] Anwar MA, Shah M, Kim J, Choi S. Recent clinical trends in toll-like receptor targeting therapeutics. Med Res Rev 2019;39(3):1053-90.

[17] Delaney TA, Morehouse C, Brohawn PZ, Groves C, Colonna M, Yao Y, et al. Type I IFNs regulate inflammation, vasculopathy, and fibrosis in chronic cutaneous graft-versus-host disease. J Immunol 2016;197(1):42-50.

[18] Shah M, Anwar MA, Kim JH, Choi S. Advances in antiviral therapies targeting tolllike receptors. Expert Opin Investig Drugs 2016;25(4):437-53.

[19] Bell J, Dymond M, Biffen M, Delaney S, Keeling D, Zhang H, et al. Temporal cytokine and lymphoid responses to an inhaled TLR7 antedrug agonist in the cynomolgus monkey demonstrates potential safety and tolerability of this approach. Toxicol Appl Pharmacol 2018;338:9-19.

[20] Wetzler LM. The role of toll-like receptor 2 in microbial disease and immunity. Vaccine 2003;21(Suppl 2):S55-60

[21] Tan ACL, Mifsud EJ, Zeng W, Edenborough K, McVernon J, Brown LE, et al. Intranasal administration of the TLR2 agonist pam2cys provides rapid protection against influenza in mice. Mol Pharm 2012;9(9):2710-8.

[22] Chua BY, Wong CY, Mifsud EJ, Edenborough KM, Sekiya T, Tan AC, et al. Inactivated influenza vaccine that provides rapid, innate-immune-system-mediated protection and subsequent long-term adaptive immunity. mBio 2015;6(6) e01024-15

[23] Mifsud EJ, Tan AC, Brown LE, Chua BY, Jackson DC. Generation of adaptive immune responses following influenza virus challenge is not compromised by pre-treatment with the TLR-2 agonist Pam2Cys. Front Immunol 2015;6:290.

[24] Enkirch T, von Messling V. Ferret models of viral pathogenesis. Virology 2015;479-480:259-70

[25] Buhnerkempe MG, Gostic K, Park M, Ahsan P, Belser JA, Lloyd-Smith JO. Mapping influenza transmission in the ferret model to transmission in humans. eLife 2015;4.

[26] Wan Y, Shang J, Graham R, Baric RS, Li F. Receptor recognition by the novel coronavirus from Wuhan: an analysis based on decade-long structural studies of SARS coronavirus. J Virol 2020;94(7).

[27] van den Brand JMA, Haagmans BL, Leijten L, van Riel D, Martina BEE, Osterhaus ADME, et al. Pathology of experimental SARS coronavirus infection in cats and ferrets. Vet Pathol 2008;45(4):551-62.

[28] Shi J, Wen Z, Zhong G, Yang H, Wang C, Huang B, et al. Susceptibility of ferrets, cats, dogs, and other domesticated animals to SARS-coronavirus 2. Science 2020;368(6494):1016-20.

[29] Ryan KA, Bewley KR, Fotheringham SA, Brown P, Hall Y, Marriott AC, et al. Dosedependent response to infection with SARS-CoV-2 in the ferret model: evidence of protection to re-challenge. bioRxiv. 2020 2020.05.29.123810.

[30] Buwitt-Beckmann U, Heine H, Wiesmüller KH, Jung G, Brock R, Akira S, et al. TLR1- and TLR6-independent recognition of bacterial lipopeptides. J Biol Chem 2006;281(14):9049-57.

[31] Buschle M, Schmidt W, Zauner W, Mechtler K, Trska B, Kirlappos H, et al. Transloading of tumor antigen-derived peptides into antigen-presenting cells. PNAS 1997;94(7):3256-61.

[32] Nguyen DT, de Witte L, Ludlow M, Yüksel S, Wiesmüller KH, Geijtenbeek TB, et al The synthetic bacterial lipopeptide Pam3CSK4 modulates respiratory syncytial virus infection independent of TLR activation. PLoS Pathog 2010;6(8):e1001049.

[33] Caly L, Druce J, Roberts J, Bond K, Tran T, Kostecki R, et al. Isolation and rapid sharing of the 2019 novel coronavirus (SARS-CoV-2) from the first patient diagnosed with COVID-19 in Australia. Med J Aust 2020;212(10):459-62.

[34] Lewandowski K, Xu Y, Pullan ST, Lumley SF, Foster D, Sanderson N, et al. Metagenomic nanopore sequencing of influenza virus direct from clinical respiratory samples. J Clin Microbiol 2019;58(1)

[35] Sungnak W, Huang N, Bécavin C, Berg M, Queen R, Litvinukova M, et al. SARSCoV-2 entry factors are highly expressed in nasal epithelial cells together with innate immune genes. Nat Med 2020;26(5):681-7.

[36] Oliveira-Nascimento L, Massari P, Wetzler LM. The role of TLR2 in infection and immunity. Front Immunol 2012;3:79. 
[37] McClure R, Massari P. TLR-dependent human mucosal epithelial cell responses to microbial pathogens. Front Immunol 2014;5:386.

[38] Banner D, Kelvin AA. The current state of H5N1 vaccines and the use of the ferret model for influenza therapeutic and prophylactic development. J Infect Develop Countries 2012;6(6):465-9.

[39] Park SJ, Yu KM, Kim YI, Kim SM, Kim EH, Kim SG, et al. Antiviral efficacies of FDAapproved drugs against SARS-CoV-2 infection in ferrets. mBio. 2020;11(3).
[40] Moreno-Fierros L, García-Silva I, Rosales-Mendoza S. Development of SARSCoV-2 vaccines: should we focus on mucosal immunity? Expert Opin Biol Ther 2020.

[41] van Doremalen N, Lambe T, Spencer A, Belij-Rammerstorfer S, Purushotham JN, Port JR, et al. ChAdOx1 nCoV-19 vaccine prevents SARS-CoV-2 pneumonia in rhesus macaques. Nature 2020;586(7830):578-82. 


\section{University Library}

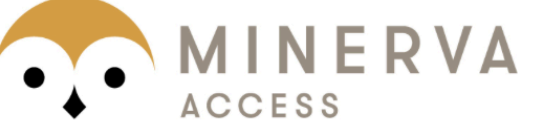

A gateway to Melbourne's research publications

Minerva Access is the Institutional Repository of The University of Melbourne

Author/s:

Proud, PC;Tsitoura, D;Watson, RJ;Chua, BY;Aram, MJ;Bewley, KR;Cavell, BE;Cobb, R;Dowall, S;Fotheringham, SA;Ho, CMK;Lucas, V;Ngabo, D;Rayner, E;Ryan, KA;Slack, GS;Thomas, S;Wand, N;Yeates, P;Demaison, C;Zeng, W;Holmes, I;Jackson, DC;Bartlett, NW;Mercuri, F;Carroll, MW

Title:

Prophylactic intranasal administration of a TLR2/6 agonist reduces upper respiratory tract viral shedding in a SARS-CoV-2 challenge ferret model

Date:

2021-01-01

Citation:

Proud, P. C., Tsitoura, D., Watson, R. J., Chua, B. Y., Aram, M. J., Bewley, K. R., Cavell, B. E., Cobb, R., Dowall, S., Fotheringham, S. A., Ho, C. M. K., Lucas, V., Ngabo, D., Rayner, E., Ryan, K. A., Slack, G. S., Thomas, S., Wand, N., Yeates, P. ,... Carroll, M. W. (2021). Prophylactic intranasal administration of a TLR2/6 agonist reduces upper respiratory tract viral shedding in a SARS-CoV-2 challenge ferret model. EBIOMEDICINE, 63, https:// doi.org/10.1016/j.ebiom.2020.103153.

Persistent Link:

http://hdl.handle.net/11343/272186

License:

CC BY-NC-ND 